\title{
Coulomb corrections in the nuclear statistical equilibrium regime
}

\author{
D. GARCíA
}

Dpt de Física i Enginyeria Nuclear, UPC, Barcelona, Spain, and Laboratori d'Astrofísica, Institut d'Estudis Catalans.

\section{E. BRAVO}

Dpt de Física i Enginyería Nuclear, UPC, Barcelona, Spain. Laboratori d'Astrofisica, Institut d'Estudis Catalans and Centre d'Estudis Avançats, C.S.I.C, Blanes, Spain

\begin{abstract}
The ionic contribution to the Equation of State (EOS) of a multicomponent plasma of nuclei in the nuclear statistical equilibrium regime is studied, and a method to compute the coulombic corrections within the framework of the linear mixing hyphothesis is proposed. Some consequences of including these corrections in the EOS are briefly analysed in relation with two concrete astrophysical scenarios, the Supernovae Ia explosion and the Accretion Induced Collapse of a massive white dwarf.
\end{abstract}

\subsection{Introduction}

Since the pioneering work of Salpeter (1961) dealing with the corrections to an ideal plasma at zero temperature non ideal effects and, specially, the Coulomb corrections have been incorporated to the EOS of stellar evolutionary codes in order to get a better understanding of the stellar evolution. The late stages of stellar evolution are specially sensitive to the non-ideal effects: the negative Coulomb pressure contributes to bound the cores of red giant stars and in white dwarfs the Coulomb and quantum corrections can drastically alter its cooling time. In the case of the progenitors of supernovae and neutron stars, the Coulomb pressure diminishes the Chandrasekhar mass limit below its nominal value of $M_{\mathrm{Ch}}=5.8 Y_{\mathrm{e}}^{2} M_{\odot}$.

The importance of Coulomb corrections in a finite temperature plasma. is measured by the plasma coupling constant, $\Gamma_{\mathrm{i}}=\left(Z_{\epsilon}\right)^{2} / r_{\mathrm{i}} k_{\mathrm{B}} T$, where $r_{\mathrm{i}}$ 
is the mean interionic distance. Because of the relative independence of the Coulomb terms with respect to the temperature, traditionally this correction has been applied to moderately cold material at high density, like that found in white dwarfs or in the cores of giant stars, being important to determine the presupernova structure. Nevertheless, the Coulomb correction has usually been neglected once the explosive thermonuclear runaway starts in the cores of the progenitors of supernovae. In view of the $Z^{5 / 3}$ dependence of $\Gamma_{i}$, the Coulomb correction to the equation of state could be important at high temperature when matter is in the nuclear statistical equilibrium (NSE).

\subsection{Coulomb corrections in NSE}

For a MCP, in the linear mixing approximation and for $\Gamma_{\mathrm{i}}>1$, the correction due to the electric interaction of the ions with an uniform electron background is given by a mean over the correction each element would have if it was the only species present (one-component plasma, OCP). For $\Gamma_{i}<1$ this assumption could not be true but in this case the corrections are not so relevant and a calculation by using an interpolation formulae (Yakovlev \&. Shalybkov 1987) probably suffice. Adopting the expression obtained by Ogata \& Ichimaru (1987) for the OCP, we have calculated the coulombic correction to the free energy per nuclei in $\mathrm{NSE}, f_{\mathrm{c}}$, from:

$$
f_{\mathrm{c}}=a \Gamma_{\mathrm{e}} \psi_{1}+4\left(\iota \Gamma_{\mathrm{e}}^{1 / 4} \psi_{2}-c \Gamma_{\mathrm{e}}^{-1 / 4} \psi_{3}\right)+d \ln \Gamma_{\mathrm{e}}-\frac{5}{3} d \psi_{4}-\epsilon,
$$

in units of $k_{\mathrm{B}} T$, being $\Gamma_{\mathrm{e}}=e^{2} / a_{\mathrm{e}} k_{\mathrm{B}} T, a_{\mathrm{e}}$ the mean interelectronic distance, $a=-0.898004, b=0.96786, c=0.220703, d=-0.86097, c=2.5269$. The $\psi_{i}$ are averages over the chemical composition,

$$
\begin{aligned}
\psi_{1}=\sum_{\nu} X_{\nu} Z_{\nu}^{5 / 3} ; & \psi_{2}=\sum_{\nu} X_{\nu} Z_{\nu}^{5 / 12} \\
\psi_{3}=\sum_{\nu} X_{\nu} Z_{\nu}^{-5 / 12} ; & \psi_{4}=\sum_{\nu} X_{\nu} \ln \left(Z_{\nu}\right)
\end{aligned}
$$

where $X_{\nu}$ is the number fraction of the species $\nu$.

In NSE the photodisintegration reactions are so fast that maintain the chemical abundances in equilibrium for densities greater than $\sim 10^{8} \mathrm{~g} / \mathrm{cm}^{3}$, once a high enough temperature $\left(\geq 510^{9} \mathrm{~K}\right)$ has been achieved. Thus, the chemical composition can be obtained as a function of $\rho, T$ and $Y_{\mathrm{e}}$ only, and the $\psi_{i}$ can be evaluated as well. We have computed the NSE abundances for a set of values of $\rho, T$, and $Y_{\mathrm{e}}\left(10^{8} \leq \rho \leq 10^{12} \mathrm{~g} / \mathrm{cm}^{3}, 210^{9} \leq T \leq 410^{10} \mathrm{~K}\right.$, and $0.40 \leq Y_{\mathrm{e}} \leq 0.50$ ), taking into account the nuclear binding energy 
and the chemical potential of each nuclei (Mochkovitch \& Nomoto 1986) corrected by the electrostatic interaction. In Fig. 1 there is plotted the pressure correction relative to the ideal gas pressure, as a function of the temperature for different densities, and for $Y_{\mathrm{e}}=0.5 \mathrm{~mol} / \mathrm{g}$.

\subsection{Specific scenarios}

\subsubsection{Supernovae Ia (SNIa)}

The ultimate effect of the coulombic corrections in NSE must be evaluated following a complete hydrodynamical calculation of a explosion model. Nevertheless, we can by now outline the main consequences on the dynamics of the explosion and the nucleosynthesis.

As can be seen in Fig. 1 the corrections in NSE are specially important at densities near $10^{10} \mathrm{~g} / \mathrm{cm}^{3}$. In the standard model of SNIa a carbon deflagration sets in at a central density $\rho \sim 3-410^{9} \mathrm{~g} / \mathrm{cm}^{3}$. Therefore, although no negligible, coulombic corrections will probably not alter too much the dynamics of the explosion.

The composition of the ejecta of SNIa is altered by the inclusion of the Coulomb chemical potentials in the Saha equation that governs the abundances of the nuclides in NSE. The negative contribution of the electrostatic interaction to the chemical potential is more important for high $Z$ nuclei, so its abundance is increased. In Fig. 2 there are compared the NSE abundances calculated with and without coulombic corrections for typical conditions at freeze-out $\left(\rho=10^{8} \mathrm{~g} / \mathrm{cm}^{3}, T=510^{9} \mathrm{~K}\right)$. The abundance of alpha particles is the most affected, being reduced by about $15 \%$ for $Y_{\mathrm{e}}=0.5 \mathrm{~mol} / \mathrm{g}$. This should reduce the amount of alpha-rich freeze-out in the expanding material of the SNIa, reducing the abundance of nuclei such as ${ }^{58} \mathrm{Ni}$ and ${ }^{62} \mathrm{Ni}$, and allowing more ${ }^{54} \mathrm{Fe}$ to be ejected to the interstellar medium. The a.bundance of neutronized nuclei can be affected in other way by reducing the net rate of electron capture in NSE. At $\rho=210^{9} \mathrm{~g} / \mathrm{cm}^{3}$ and $T=810^{9} \mathrm{~K}$ (typical conditions of the material when most of the electron captures take place) the main contribution to the neutronization comes from the Co isotopes. In Fig. 3 there are shown the variation of the Co isotopes abundances, due to the inclusion of the Coulomb chemical potentials. The differences can reach $\sim 40 \%$, and a similar increase in the neutronization rate can be expected (except for the important contribution of free protons, whose abundance is nearly unaffected). 


\subsubsection{Accretion Induced Collapse (AIC)}

For certain cases, the central ignition of a massive white dwarf destabilized by matter accretion from a companion can take place at densities as high as $10^{10} \mathrm{~g} / \mathrm{cm}^{3}$ (Hernanz et al. 1988). At such a high density the electron captures onto the nuclei present in the NSE regime can be fast enough to induce the collapse, rather than the explosion, of the white dwarf, forming a neutron star. Then, for a fixed initial chemical composition of the white dwarf (Carbon and Oxygen, or Oxygen-Neon-Magnesium) there does exist a critical density which separates the explosive and collapsing outcome. The value of this density is a function of the velocity of the conductive burning front that determines the advance of the flame near to the center. As a general rule, slow fronts lead to collapse and fast fronts lead to explosion.

Recently, Timmes \&. Woosley (1992) have calculated the velocity of a conductive burning front as a function of the chemical composition prior to ignition, and of the ignition density. When this expression for the conductive velocity is used to follow the evolution of a massive white dwarf without taking into account the coulombic corrections, a value of $\rho_{\mathrm{c}} \simeq 8.510^{9} \mathrm{~g} / \mathrm{cm}^{3}$ is obtained for the critical density. However:

- Coulombic effects alter the velocity of the conductive combustion front itself. In order to obtain an idea of the importance of non-ideal corrections on the conductive velocity, the hydrodynamic evolution of a conductive flame in a medium composed of $\mathrm{C}$ and $\mathrm{O}$ has been followed by means of an hydrocode, in the form described in Garcia et al. (1990), and using a small nuclear network to follow the changes in the elemental abundances. Although a larger network is needed for a suitable representation of the flame velocity (Timmes \&. Woosley 1992), our approach is sufficient to compare the effect of including the coulombic correction. Our calculations show that an overestimation of the flame velocity in about $10 \%$ results when the specific heat $c_{\mathrm{v}}$ is not corrected from non ideal terms.

- Near $\rho=10^{10} \mathrm{~g} / \mathrm{cm}^{3}$ the overpressure caused by the almost instantaneous rise in temperature is about $10 \%$, and it is greatly due to the ionic contribution. In these conditions a coulombic correction of about $35 \%$ (see curve $c$ in Fig. 1) represents a substantial reduction in the total overpressure.

In Fig. 4 there is shown the evolution of a white dwarf after the explosive carbon ignition at $\rho_{c}=710^{9} \mathrm{~g} / \mathrm{cm}^{3}$. Curve (1) represents the evolution obtained using an EOS without coulombic corrections in the NSE regime, and with the conductive burning velocity given by Timmes \& Woosley. Curve (2) is the same, but including coulombic corrections in NSE, and with the 
front velocity multiplied by a factor 0.9 , according with the above mentioned results. As can be seen, the track of the central density is substantially altered, changing from explosion to collapse for the same ignition density.

\subsection{Conclusions}

A simple method to compute the Coulomb corrections in a complex system composed of several hundred of nuclei which are in nuclear statistical equilibrium has been presented. The main implications for SNIa models are of nucleosynthetic nature: the rate of neutronization at a fixed temperature can be increased by $\sim 40 \%$ in the heavy nuclei contribution at $Y_{\mathrm{e}}=0.50 \mathrm{~mol} / \mathrm{g}$, and the amount of alpha-rich freeze-out reduced by about $15 \%$.

The AIC is favoured by the pressure decrease and specific heat increase due to the Coulomb terms. This fact, besides a slight reduction on the conductive velocity of the thermonuclear front, allows the minimum density at which a C-O white dwarf could collapse be as low as $710^{9} \mathrm{~g} / \mathrm{cm}^{3}$.

This work has been supported by the D.G.I.C.Y.T. grant PB90-0912, and the C.E.S.C.A. project Hydrodynamical Evolution of Compact S'tar's.

\section{References}

Garcia, D., Labay, J., Canal, R. and Isern, J., Proceedings of the Symposium

"Nuclei In The Cosmos", Baden (Austria), Eds H. Oberhummer and W.

Hillebrandt, p97, 1990.

Hernanz, M., Isern, J., Canal, R. and Labay, J.,ApJ, 324, p331, 1988.

Mochkovitch, R. and Nomoto, K., Astronomy and Astrophysics, 154, p115, 1986.

Ogata, S. and Ichimaru, S., Physical Revieu, A36, p5451, 1987.

Salpeter, E., $A p J$, 134, p669, 1961.

Timmes, F.K. and Woosley, S.E., Ap J, 396, p649, 1992.

Yakovlev, D.G. and Shalybkov D.A.,Sov. Sci. Rev. E. Astrophysics and Space Physics, 7, p311, 1989.

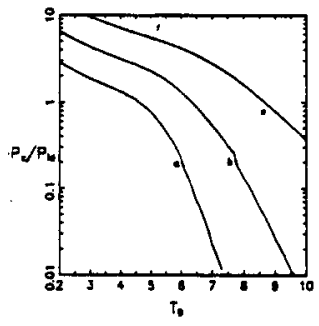

Figure 1. Prowaure correction relotive to

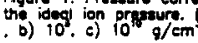

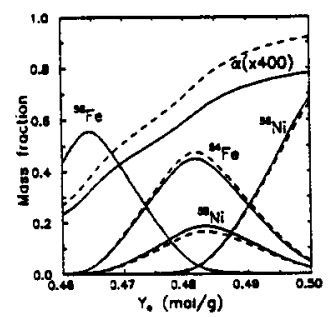

Figure 2. Abundances in NSE at freez out. With Coulome enemicol potentials
(solid line) ond witheut (datined line)

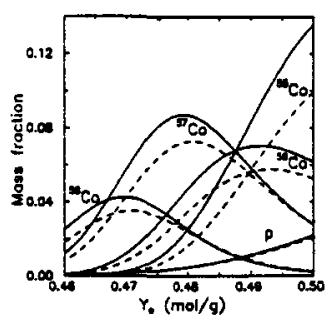

Figure 3. Abunadeneses of the main (dosned line) Coulomb and without

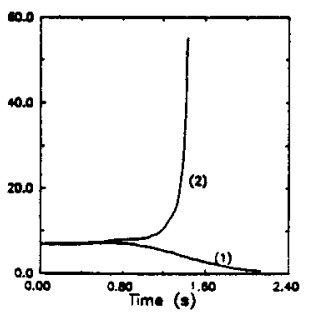
Figure 4. Evolution of $\rho_{3}$ for, $0 \mathrm{C}-\rho$ 